\title{
The influence of perivascular adipose tissue on vascular homeostasis
}

This article was published in the following Dove Press journal:

Vascular Health and Risk Management

20 March 2013

Number of times this article has been viewed

\author{
Theodora Szasz' \\ Gisele Facholi Bomfim² \\ R Clinton Webb' \\ 'Department of Physiology, Georgia \\ Regents University, Augusta, USA; \\ ${ }^{2}$ Department of Pharmacology, \\ University of São Paulo, \\ São Paulo, Brazil
}

Correspondence: Theodora Szasz Department of Physiology, Georgia Regents University, I I 20 I5th St, Augusta, GA 30912, USA

$\mathrm{Tel}+\mid \mathrm{I} 70672$ I 3547

Fax +I 7067217299

Email iszasz@gru.edu
Abstract: The perivascular adipose tissue (PVAT) is now recognized as an active contributor to vascular function. Adipocytes and stromal cells contained within PVAT are a source of an ever-growing list of molecules with varied paracrine effects on the underlying smooth muscle and endothelial cells, including adipokines, cytokines, reactive oxygen species, and gaseous compounds. Their secretion is regulated by systemic or local cues and modulates complex processes, including vascular contraction and relaxation, smooth muscle cell proliferation and migration, and vascular inflammation. Recent evidence demonstrates that metabolic and cardiovascular diseases alter the morphological and secretory characteristics of PVAT, with notable consequences. In obesity and diabetes, the expanded PVAT contributes to vascular insulin resistance. PVAT-derived cytokines may influence key steps of atherogenesis. The physiological anticontractile effect of PVAT is severely diminished in hypertension. Above all, a common denominator of the PVAT dysfunction in all these conditions is the immune cell infiltration, which triggers the subsequent inflammation, oxidative stress, and hypoxic processes to promote vascular dysfunction. In this review, we discuss the currently known mechanisms by which the PVAT influences blood vessel function. The important discoveries in the study of PVAT that have been made in recent years need to be further advanced, to identify the mechanisms of the anticontractile effects of PVAT, to explore the vascular-bed and species differences in PVAT function, to understand the regulation of PVAT secretion of mediators, and finally, to uncover ways to ameliorate cardiovascular disease by targeting therapeutic approaches to PVAT.

Keywords: adipokines, vascular dysfunction

\section{Perivascular adipose tissue (PVAT) anatomy}

PVAT is, by definition, situated outside of the blood vessel and is structurally distinct from the adventitia, although no clear barrier exists between the two. Depending on the anatomical region and vessel caliber, PVAT may be more abundant (as with the aorta), debatably separated from the surrounding adipose tissue (for eg, the coronary PVAT within epicardial fat), or often absent (cerebral or microcirculation). In clinical studies that categorize adipose tissue as either subcutaneous (SAT) or visceral (VAT), the PVAT around large arteries, such as the aorta or mesenteric arteries, is generally grouped in together with VAT, famously correlated with cardiovascular risk. This classification is supported by studies quantifying aortic PVAT mass by computed tomography (CT)-based volumetric measurements, which demonstrated a strong correlation between aortic PVAT and VAT. ${ }^{1}$ Conversely, seminal epidemiological studies that identified aortic PVAT separately from VAT found that the volume of this specific kind of visceral fat, just as of VAT per se, correlated with hypertension, 
diabetes, and aortic and coronary calcification, even if corrected for body mass index. ${ }^{2}$

In terms of its classification as an adipose tissue, PVAT is not necessarily white adipose tissue (WAT) or brown adipose tissue (BAT). Thus, there are instances of both white and mixed PVAT, such as the rodent mesenteric and aortic PVAT, respectively. This mixed aortic PVAT resembles more the classical BAT, ${ }^{3}$ with multilocular adipocytes abundant in mitochondria and expressing uncoupling protein-1 (UCP-1), whereas mesenteric PVAT is white in nature, with larger unilocular adipocytes that are lacking UCP-1 and comparatively less vascularized. The traditional roles ascribed to WAT, as a lipid deposit with little metabolic activity, and to BAT, as a site of nonshivering thermogenesis, are nowadays insufficient in describing the rich endocrine activity of both types of tissue and indeed, of PVAT as well, which due to this activity, is deeply involved in the function of the blood vessels it surrounds.

PVAT differs substantially from other fat depots with respect to its secretory profile. For example, mouse aortic PVAT produces less adiponectin, leptin, and resistin, expresses lower levels of lipid-oxidation genes, and has the reverse expression profile of adipose-related and lipid synthesis and storage genes compared with SAT and VAT., Comparatively, transcriptome analyses have shown there are far less differences in gene expression between murine aortic PVAT and interscapullary BAT, nominally only a total of 228 genes, while registering similar expression levels for classically brown adipocyte-enriched genes, such as UCP-1 and Cidea. ${ }^{3}$ The secretory profile is not the only characteristic distinguishing the PVAT from SAT or VAT. Markers of adipocyte differentiation and maturation, such as lipoprotein lipase, glycerol phosphate 3 dehydrogenase, or perilipin, have a relative decreased expression in PVAT compared with SAT and VAT. ${ }^{4}$ The two studies cited above differ in one key aspect and that is the expression of immune and inflammatory genes. In one study, genes like interleukin (IL)-6, IL-8, or monocyte chemoattractant protein 1 (MCP1) were higher in PVAT than in SAT and VAT, ${ }^{4}$ whereas in the other, chemokines, T-cell receptor and macrophage markers had lower PVAT expression. ${ }^{3}$ Consequently, the inflammatory alterations that occurred under high fat diet (HFD) conditions were either more ${ }^{4}$ or less ${ }^{3}$ severe in PVAT, aspects which are discussed later. In yet another study, the secretion of cytokines, angiogenic factors, and metabolic hormones was measured in cultured adipocytes from human SAT, VAT, and radial artery PVAT. Results demonstrated that the PVAT adipocytes secretion pattern was different from that of the other fat deposits, having a higher production of angiogenic factors and cytokines, like hepatocyte growth factor (HGF), vascular endothelial growth factor, MCP-1, and thrombospondin $1 .{ }^{5}$ Coculture of these adipocytes with endothelial cells increased production of MCP-1 and granulocyte colony stimulating factor. HGF, previously identified as an adipokine, ${ }^{6}$ was preferentially released from PVAT adipocytes and induced VSMC cytokine release and endothelial cell proliferation. Importantly, magnetic resonance imaging (MRI)-determined PVAT mass, but not mass of SAT or VAT, correlated with circulating HGF, in patients with increased risk for diabetes, thus highlighting $\mathrm{HGF}$ as a potential marker of PVAT mass in humans. ${ }^{5}$

Consequent to the structural differences in the PVAT of various vascular beds, there are secretory-profile and adipose gene-expression differences, similar to the ones between WAT and BAT. For instance, a study comparing expression of renin-angiotensin system components between mesenteric and aortic PVAT in Wistar Kyoto (WKY) rats demonstrated not just the predictable morphological differences derived from the white-like mesenteric PVAT or the brown-like aortic PVAT, but also higher angiotensin $\mathrm{AT}_{1 \mathrm{a}}{ }^{-}$and $\mathrm{AT}_{2}-$ receptor, chymase, and angiotensin II expression, and lower prorenin-receptor expression in mesenteric compared with aortic PVAT. $^{7}$ The point that not all PVAT is structurally the same is one to bear in mind when comparing results from PVAT studies, not all of which were performed in the same vascular bed.

Adipocytes are the most abundant cell population in PVAT. Although morphologically resembling white or brown adipocytes, according to the particular vascular bed they surround, these cells may not have the same developmental origin as their subcutaneous or visceral counterparts. The very idea of strictly defining adipocytes as white or brown, based on only mitochondrial content or UCP-1 expression, has come under intense scrutiny recently. This separation does not take into account the presence of brown-like adipocytes occurring in the midst of a white adipose tissue, such as after cold exposure, treatment with peroxisome proliferatoractivated receptor (PPAR) $\gamma$ - or $\beta_{3}$-adrenergic agonists, or in PPAR $\gamma$ coactivator 1 overexpression, instances that support the idea of white-to-brown transdifferentiation or white preadipocyte neodifferentiation. ${ }^{8}$ The reverse brown-to-white transformation is also a common phenomenon during the development of larger mammals, including humans. Given the emerging concept that BAT is inherently beneficial and WAT promotes inflammation, efforts are currently directed at finding the factors that would induce WAT "browning" 
and the formation of "beige" or "brite" adipose tissue. ${ }^{9}$ In this context, it is noteworthy that exercise promotes PPAR $\gamma$ coactivator 1 expression in skeletal muscle and induces irisin secretion, which in turn stimulates white-to-brown developmental programs in white adipocytes.$^{10}$ Exercise, in an enriched housing environment, also induced the activation of a theorized hypothalamic-adipocyte axis in rodents that led in turn, to a white-to-brown phenotypic transformation, potentially mediated by brain-derived neurotrophic factor overexpression in the hypothalamus. ${ }^{11}$

Besides adipocytes, PVAT contains other cells grouped together as the stromal vascular fraction (SVF), including fibroblasts, mesenchymal stem cells, lymphocytes, macrophages, and potentially, endothelial cells from accompanying structures, such as the vasa vasorum. The SVF cells, due to their diverse properties and functions, are of extreme importance in determining the release of vasoactive or other biologically relevant substances from PVAT (Figure 1). Furthermore, in disease conditions marked by an increase in the number of SVF cells in the PVAT, via mechanisms such as macrophage infiltration, this fraction becomes even more relevant.

The extracellular matrix (ECM) of PVAT is similar to that of other adipose tissues and contains collagen, laminin, and fibronectin fibers. Adipose ECM is under continuous dynamic remodeling by ECM-processing enzymes, such as the matrix metalloproteinases, tissue inhibitors of metalloproteinases, and proteins from the a disintegrin and metalloproteinase with thrombospondin motifs (ADAMTS) family. ${ }^{12}$

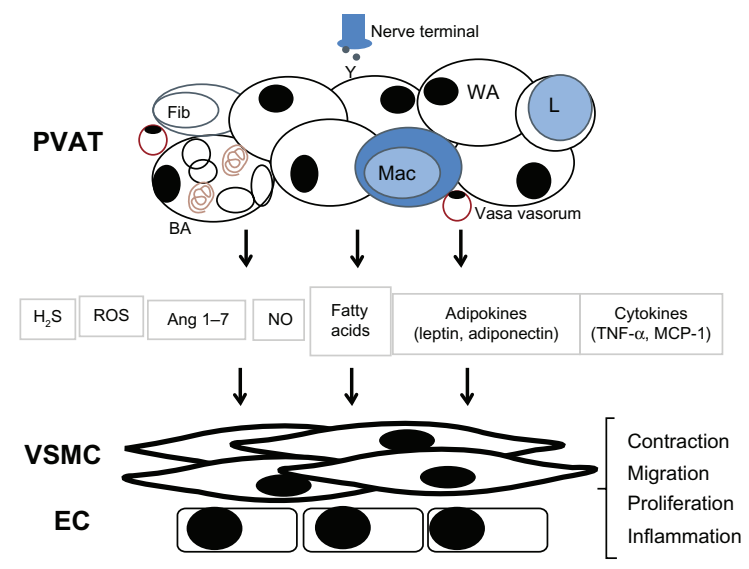

Figure I Perivascular adipose tissue releases molecules that modulate vascular function.

Abbreviations: Ang I-7, angiotensin I-7; BA, brown adipocyte; EC, endothelial cell; Fib, fibroblast; $\mathrm{H}_{2} \mathrm{~S}$, hydrogen sulfide; L, lymphocyte; Mac, macrophage; MCP-I, monocyte chemoattractant protein-I; NO, nitric oxide; PVAT, perivascular adipose tissue; ROS, reactive oxygen species; TNF $\alpha$, tumor necrosis factor $\alpha$; VSMC, vascular smooth muscle cell; WA, white adipocyte; $Y$, autonomic nervous system receptor.
PVAT also contains sympathetic nervous fibers and, depending on vascular bed, may also contain vasa vasorum. Parasympathetic innervation of WAT has been ruled out; $;^{13}$ however, no retrograde labeling studies have been performed specifically on PVAT. The control of PVAT function via sympathetic innervation is a recent concept and part of the previously mentioned complex concept of a brain-adipocyte axis, which warrants further study, especially given the alterations observed during disease states. ${ }^{14}$

\section{PVAT function}

\section{Release of adipokines and other bioactive molecules}

Adipose tissue was, until recently, seen as mere connective tissue supporting the adjacent structures, with additional functions in lipid deposition (WAT) and nonshivering thermogenesis (BAT). Conversely in vascular biology, PVAT was seen as providing vascular support, and as it was thought to impede diffusion of exogenous molecules, it was (and still is, for the most part) removed for vascular function studies. However, compelling evidence from the last two decades has led to the wide acceptance of adipose tissue, in general, as an important endocrine organ and of PVAT, in particular, as having notable paracrine effects on blood vessels. These effects, discussed below, are mediated by a host of molecules (Table 1) that are released from the various PVAT cell populations.

Perhaps the most important category of substances released from PVAT is that of the adipokines, termed as such because of their adipocyte origin and cytokine-like effects. Of these, adiponectin and leptin have been extensively studied. Adiponectin was first identified in $1995,{ }^{15}$ and although an adipocyte-specific product, it was soon observed that its levels are decreased in obesity, ${ }^{16}$ a discovery that prompted the initiation of numerous studies. Adiponectin is a small protein of $30 \mathrm{kDa}$ in its globular form. Its collagenous $\mathrm{N}$-terminal domain allows for formation of multimers that bind to the adiponectin receptors ADIPOR1 and ADIPOR2 for intracellular signaling. Several additional proteins, such as T-cadherin have been implicated in mediating adiponectin effects. ${ }^{17}$ These effects are complex and range from promoting pancreatic beta cell survival and insulin secretion as well as insulin sensitivity, renal, and cardiac protection, to important local autocrine and systemic anti-inflammatory effects. ${ }^{18-21}$ Blood vessel function may be modulated by adiponectin through various mechanisms, including stimulation of vasorelaxation, ${ }^{22}$ adenosine monophosphate-activated 
Table I Substances released by PVAT, their effects on normal vascular function, and alterations during PVAT dysfunction in cardiovascular disease

\begin{tabular}{|c|c|c|}
\hline Produced by PVAT & Effect on normal vascular function & PVAT dysfunction \\
\hline \multirow[t]{5}{*}{ Leptin } & Direct vasodilatory effect ${ }^{24-28}$ & Increased PVAT production in obesity $4,39,40$ \\
\hline & Increases VSMC proliferation/migration ${ }^{69,70}$ & Decreased PVAT production in hypertension ${ }^{89}$ \\
\hline & Decreases VSMC proliferation ${ }^{29,71}$ & Effects on VSMC contraction lost in hypertension ${ }^{29,95}$ \\
\hline & Increases vascular permeability ${ }^{32}$ & Increased PVAT production in atherosclerosis ${ }^{35,99-103,105}$ \\
\hline & Participates in PVAT anticontractile effect ${ }^{61}$ & \\
\hline \multirow[t]{4}{*}{ Adiponectin } & Direct vasodilatory effect ${ }^{22,23}$ & Decreased PVAT production in obesity $4,39,40$ \\
\hline & Decreases VSMC proliferation/migration ${ }^{73-75}$ & Decreased PVAT production in diabetes ${ }^{87}$ \\
\hline & Participates in PVAT anticontractile effect ${ }^{22,56,59}$ & Decreased production in atherosclerosi $\mathrm{s}^{35,99-103}$ \\
\hline & Anti-inflammatory; protects from endothelial injury ${ }^{20}$ & \\
\hline Resistin & Increases VSMC proliferation/migration ${ }^{68}$ & Increased in endothelial-injury model ${ }^{68}$ \\
\hline Visfatin & Increases VSMC proliferation/migration ${ }^{65}$ & Increased PVAT production in atherosclerosis ${ }^{35,99-103,105}$ \\
\hline HGF & Induces endothelial cell proliferation ${ }^{5}$ & Increased PVAT production in obesity.6 \\
\hline Other adipokines (nesfatin, & Chemerin increases VSMC contraction ${ }^{62}$ & Increased PVAT production in atherosclerosis ${ }^{105}$ \\
\hline adrenomedulin, vaspin, & Omentin has direct vasodilatory effect ${ }^{33}$ & \\
\hline omentin, chemerin, adipsin) & Potential participation in PVAT anticontractile effects ${ }^{63}$ & \\
\hline \multirow[t]{3}{*}{ TNF $\alpha$} & & Correlated with PVAT inflammation and hypoxia \\
\hline & & in human obesity ${ }^{59}$ \\
\hline & & Increased PVAT production in atherosclerosis ${ }^{35,99-103}$ \\
\hline Interleukins (IL-I, IL-6, IL-8) & & Increased PVAT production in atherosclerosis ${ }^{35,99-103,105}$ \\
\hline \multirow[t]{2}{*}{ MCP-I } & & Increased PVAT production in atherosclerosis ${ }^{35}$ \\
\hline & & Increased PVAT production in obesity ${ }^{39}$ \\
\hline 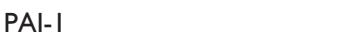 & Increases VSMC proliferation & Increased PVAT production in atherosclerosis ${ }^{35,99-103}$ \\
\hline Reactive oxygen species & Superoxide may promote procontractile PVAT effects ${ }^{37}$ & Increased PVAT production in obesity ${ }^{39,40,59,82}$ \\
\hline (superoxide, $\mathrm{H}_{2} \mathrm{O}_{2}$ ) & $\mathrm{H}_{2} \mathrm{O}_{2}$ participates in PVAT anticontractile effect ${ }^{36,38}$ & \\
\hline \multirow[t]{2}{*}{ Hydrogen sulfide $\left(\mathrm{H}_{2} \mathrm{~S}\right)$} & Direct vasodilatory effect ${ }^{44-46}$ & \\
\hline & Participates in PVAT anticontractile effect ${ }^{44,46}$ & \\
\hline Nitric oxide (NO) & Participates in PVAT anticontractile effect ${ }^{36}$ & Increased PVAT production in early obesity ${ }^{42}$ \\
\hline \multirow[t]{2}{*}{ Angiotensin II } & Participates in perivascular nerve & Increased PVAT production in hypertension ${ }^{34}$ \\
\hline & stimulation-induced contraction ${ }^{66}$ & \\
\hline Angiotensin (I-7) & Participates in PVAT anticontractile effect ${ }^{47,55,57,60}$ & Decreased production in hypertension ${ }^{94}$ \\
\hline \multirow[t]{2}{*}{ Methyl-palmitate } & Methyl palmitate participates in PVAT & Decreased PVAT production of methyl palmitate \\
\hline & anticontractile effect ${ }^{34}$ & in hypertension ${ }^{34}$ \\
\hline \multirow[t]{2}{*}{ Other fatty acids } & Oleic acid increases VSMC proliferation ${ }^{73}$ & FA composition in PVAT changed in \\
\hline & & metabolic syndrome ${ }^{82}$ \\
\hline Complement 3 & Induces fibroblast migration ${ }^{72}$ & Increased PVAT production in hypertension ${ }^{72}$ \\
\hline
\end{tabular}

Abbreviations: FA, fatty acid; $\mathrm{H}_{2} \mathrm{O}_{2}$, hydrogen peroxide; HGF, hepatic growth factor; IL, interleukin; MCP-I, monocyte chemoattractant protein-I; PAI-I, plasminogen activator inhibitor-I; PVAT, perivascular adipose tissue; TNF $\alpha$, tumor necrosis factor $\alpha$; VSMC, vascular smooth muscle cell.

protein kinase (AMPK)-mediated endothelial nitric oxide (NO) synthase (eNOS) activation, ${ }^{23}$ protection from endothelial injury, ${ }^{20}$ and anti-inflammatory actions.

Leptin is another essential adipocyte-specific hormone, secreted principally by adipocytes from WAT and functioning to signal energy status and regulate appetite through hypothalamic leptin-receptor signaling. Its circulating levels are proportional to the adipose tissue mass, thus a main characteristic of most obesity types is hyperleptinemia. However, due to complex mechanisms, the hyperleptinemia observed in human obesity is associated with peripheral leptin resistance, similar to the hyperinsulinemic and insulin resistant state encountered in diabetes. Similarly to adiponectin, leptin plays other important roles systemically, such as modulating the endocrine hypothalamic-pituitary signals to the gonads, thyroid, and the adrenal gland, as well as modulating insulin sensitivity, bone metabolism, and immune system function. In the cardiovascular system, where the six leptin receptors are also expressed, the effects of leptin are generally those of vasodilation, producing hypotension upon acute in vivo infusion and exerting direct vasodilatory effects on various vascular beds. ${ }^{24-26}$ Leptin-induced vasorelaxation may be mediated by endothelial NO-dependent or independent mechanisms. ${ }^{26-28}$ A VSMC inducible NO synthase (NOS)mediated anticontractile effect was also described for angiotensin II-induced contraction in rat aorta. ${ }^{29}$ However, mimicking chronic obese states, long-term infusion of leptin promotes hypertension and endothelial dysfunction. ${ }^{30,31}$ Interestingly, leptin may increase vascular permeability, and ob/ob mice that lack leptin also lack adipose tissue 
fenestrations, a feature that makes adipose tissue of normal mice resemble an endocrine tissue. ${ }^{32}$

There is by now an impressive list of other adipokines (Table 1) that each may contribute to vessel function in some way. Recently, it was shown that omentin induces vasorelaxation. ${ }^{33}$ As previously mentioned, HGF induces endothelial cell proliferation and VSMC cytokine production. ${ }^{5}$ PVAT adipocytes also release various fatty acids that may act as independent signaling molecules, such as methyl palmitate. ${ }^{34}$ Due to the presence of SVF cells, secretion of a wide array of cytokines was also demonstrated in PVAT, including ILs and tumor necrosis factor $\alpha(\mathrm{TNF} \alpha)$ as well as various angiogenic and chemotactic factors. ${ }^{3-5,35}$ In addition, much similar to the adventitial layer, PVAT expresses nicotinamide adenine dinucleotide phosphate (NADPH)oxidases, is capable of producing reactive oxygen species (ROS), like superoxide and hydrogen peroxide $\left(\mathrm{H}_{2} \mathrm{O}_{2}\right)$, and is also equipped with antioxidant defense enzymes. ${ }^{36-40}$ PVAT cells express NOS isoforms and release NO, although the exact cell type responsible for this NO production is not yet clear. ${ }^{41-43}$ The production of gaseous molecules, like hydrogen sulfide $\left(\mathrm{H}_{2} \mathrm{~S}\right)$, from PVAT has also received attention recently, especially due to the vasodilator effect of $\mathrm{H}_{2} \mathrm{~S} .{ }^{44-46}$ Other important vasoactive molecules secreted from PVAT belong to the renin-angiotensin system, including angiotensin II and angiotensin (1-7), the latter having been demonstrated as being partly responsible for the anticontractile effect of PVAT. ${ }^{7,47}$ Unlike with adipokines, the secretion of the vast majority of these molecules, when measured from whole tissue, cannot be attributed to any particular cell type.

\section{Anticontractile effects of PVAT}

The view of the PVAT as a supportive structure with no role in vascular function was the widely held belief until recent years, and the first study to challenge this came in 1991, when Soltis and Cassis ${ }^{48}$ reported that PVAT inhibited rat aorta contractile response to norepinephrine. Subsequently, Gollasch et al pioneered investigation of this phenomenon $^{22,49-52}$ and provided additional proof that PVAT inhibited contractile response to a number of additional agonists, like angiotensin II, serotonin, endothelin-1, or phenylephrine. The fact that these various agonists are all subject to inhibition by PVAT precludes the initial suggestion that PVAT would increase the uptake of norepinephrine, ${ }^{48}$ as other agonists would not be taken up, cleaved, or inactivated. Furthermore, this anticontractile effect is not due to the physical presence of PVAT mechanically impeding or decreasing the diffusion of exogenous molecules, since the supernatant from incubated
PVAT or adipocytes reproduces the effect, ${ }^{50}$ essentially demonstrating that factors released from PVAT mediate the decrease in contractile responses. It also does not appear to be a species effect, as similar results were observed in rat, mouse, dog, pig, and human vessels. This occurs in various vascular beds, like the aorta, the mesenteric circulation, coronary arteries, ${ }^{53}$ or limb vessels,${ }^{54}$ therefore suggesting it does not depend either on the white- or the brown-like quality of the adipose tissue. The anticontractile effect of PVAT was also reproduced for veins, ${ }^{55}$ which like arteries, are surrounded by PVAT.

The identity of the factor(s) that mediate the anticontractile effect of PVAT is still under discussion, but several molecules have been proven to at least partially explain it. Initially termed adventitia-derived relaxing factor, by Lohn et al, ${ }^{50}$ to mimic its endothelial counterpart, the perivascular-derived relaxing factor (PVRF) is clearly not a singular entity, as discussed below. Similar to the endothelium that does not only produce NO, PVAT is also a complex tissue and its effects cannot be reduced to any molecule alone, especially considering the impressive list of potential candidates for any effect (Table 1).

Several key observations have been made by the groups of Gollasch ${ }^{49-52}$ and Lee. ${ }^{36}$ First, as previously mentioned, the PVAT anticontractile effect is transferrable (a solution that was transferred from an incubated PVAT-intact vessel induced anticontractile effect in a separate PVAT-free vessel) and adipocyte-derived (the supernatant from cultured adipocytes reproduced these effects). ${ }^{50}$ Studies have also demonstrated the essential role played in mediating the PVAT anticontractile effect by the gated potassium $(\mathrm{K})$ channels, namely the adenosine triphosphate (ATP) channels $\left(\mathrm{K}_{\text {ATP }}\right)^{49}$ and later, the voltage-gated $\left(\mathrm{K}_{\mathrm{V}}\right)^{51}$ channels, and excluded others, like the large conductance calcium-activated $\left(\mathrm{BK}_{\mathrm{Ca}}\right)$ channels and the delayed rectifying and the inwardly rectifying channels. ${ }^{49}$ However, the role of $\mathrm{BK}_{\mathrm{Ca}}$ channels in mediating anticontractile PVAT actions was recently revealed. ${ }^{56}$ Part of the anticontractile effect is endothelium dependent, mediated by NO and calciumactivated channel $\mathrm{K}_{\mathrm{Ca}}$ activation, and part is endothelium independent, potentially mediated by $\mathrm{H}_{2} \mathrm{O}_{2}$ release and soluble guanlyate cyclase (sGC) activation. ${ }^{36} \mathrm{~K}_{\mathrm{Ca}}$ channels have also been found to be involved in mediating PVAT-induced relaxation in the human internal mammary artery. ${ }^{43,57}$

The very mechanism of release of factors from PVAT was also investigated. This release appears to be calcium ion $\left(\mathrm{Ca}^{2+}\right)$ dependent, and PKA and tyrosine kinase regulated, while independent of nerve-ending influences, as vanilloid, cannabinoid, and calcitonin gene-related peptide inhibition 
did not modify the anticontractile effect. ${ }^{49}$ The involvement of proteinase-activated receptor- $2\left(\mathrm{PAR}_{2}\right)$ was recently investigated, using its ligands or trypsin, in mouse aorta. ${ }^{38}$ Release of PVRFs may be $\mathrm{PAR}_{2}$ dependent, mediated by $\mathrm{H}_{2} \mathrm{O}_{2}$, but not sGC, different from Gao et al, ${ }^{36}$ or $\mathrm{PAR}_{2}$ independent, mediated through $\mathrm{K}_{\mathrm{V}}$ channels. ${ }^{38}$ This latter work also suggested that PVAT-derived proteinases, such as adipsin, may modulate PVAT effects on contraction. The anticontractile effect of PVAT was also inhibited or lost in the presence of mineralocorticoids, like deoxycorticosterone in the rat aorta ${ }^{48}$ or aldosterone in mesenteric arteries, where mineralocorticoid-receptor antagonists restored this effect. ${ }^{58}$

A few individual molecules have been implicated in the mediation of the relaxant or anticontractile effects of PVAT. Recombinant adiponectin has been found to reduce serotoninmediated contraction of rat aorta. ${ }^{22}$ Supporting this finding, a fragment of the adiponectin receptor was found to block the PVAT-mediated relaxation in human small arteries. ${ }^{59}$ However, reports from adiponectin-knockout mice are contradictory, the anticontractile effects of PVAT on serotonin-induced contraction being preserved in the aorta and mesenteric bed in one case, ${ }^{22}$ while being lost in the case of norepinephrineinduced contraction in small mesenteric arteries in another. ${ }^{56}$ Angiotensin (1-7) is expressed and released from PVAT and is partly responsible for the anticontractile effect, since inhibition of Mas, the receptor for angiotensin (1-7), partly blocks this effect in both rat aorta ${ }^{60}$ and rat vena cava, ${ }^{55}$ effects confirmed by similar experiments done with Mas receptor knockout mice. ${ }^{47}$ More recently, a study showed that methyl palmitate is released from rat aorta PVAT in a $\mathrm{Ca}^{2+}$-dependent manner and mediates vasorelaxation via $\mathrm{K}_{\mathrm{V}}$ channels. ${ }^{34} \mathrm{H}_{2} \mathrm{~S}$, produced in PVAT largely by the action of cystathionine- $\gamma$ lyase, is also directly involved in mediating PVAT effects on vascular reactivity, ${ }^{44,46}$ and this effect occurs via $\mathrm{KCNQ} \mathrm{K}_{\mathrm{V}}$ channels in rat, but not in mouse, as recently revealed. ${ }^{45}$ Interestingly, it appears that statins increase $\mathrm{H}_{2} \mathrm{~S}$ production, likely through inhibition of its mitochondrial oxidation. ${ }^{46}$ PVAT-released leptin was recently also demonstrated to mediate the vasorelaxant effects of PVAT. ${ }^{61}$ Other molecules from the ever-growing list of adipokines (including resistin, omentin, vaspin, nesfatin, and adipsin) may also contribute to these effects via direct vasodilatory or other mechanisms $;^{33,38,62,63}$ however, definitive studies are still lacking.

Other cell types, besides adipocytes, may mediate the vasorelaxant effects of PVAT, and one important study used a conditional macrophage ablation mouse model to prove that infiltrating macrophages were essential for PVAT function. ${ }^{58}$ These authors also indicated hypoxia as abolishing the vasorelaxant effects of PVAT, which were restored by aldosterone receptor antagonism. Contrasting with these results, Maenhaut et $\mathrm{al}^{64}$ showed that hypoxia enhanced the anticontractile effects of mouse aortic PVAT via $\mathrm{K}_{\mathrm{ATP}}$ and endothelium-independent mechanisms.

Other studies effectively ruled out the contribution of different molecules in mediating vasorelaxation by PVAT, such is the case of visfatin, ${ }^{65}$ while the opposite procontractile effect of PVAT was cited in a few studies. Gao et al ${ }^{37}$ showed that rat superior mesenteric contraction induced by perivascular nerve stimulation was mediated by superoxide release from NADPH oxidase, expressed in PVAT adipocytes. Authors in the same group demonstrated that this effect was blocked by angiotensin converting enzyme inhibitors or $\mathrm{AT}_{1}$ receptor antagonists, suggesting that PVAT-derived angiotensin II mediates the perivascular nerve stimulation-induced contraction. ${ }^{66}$ Contrasting with most results reviewed here, a study of canine coronary arteries suggested that PVAT decreases endothelium-dependent relaxation, through phosphorylation of eNOS at the Thr-495 inhibitory site by protein kinase $\mathrm{C} \beta$ (PKC $\beta){ }^{67}$

Another factor that might explain the procontractile effects of PVAT in physiological conditions besides superoxide is chemerin, an adipokine that may be produced in PVAT and that was recently demonstrated to increase phenylephrine and endothelin-induced contraction, in the absence of PVAT, via activation of mitogen-activated protein kinase (MAPK) kinase and extracellular signal-regulated kinase 1 and 2 $\left(\right.$ ERK1/2). ${ }^{62}$

\section{Effects of PVAT on proliferation and migration of VSMCs}

Besides its inhibitory effect on acute blood vessel contraction, PVAT is also involved in the proliferation and migration of VSMC, functions it generally stimulates. Adipokines and other adipose-derived factors typically increase VSMC proliferation, such is the case of resistin and visfatin. Visfatin levels were shown to be higher in PVAT than SAT and VAT. Visfatin stimulates nicotinamide mononucleotide production, which in its turn triggers VSMC growth through the insulin-independent activation of ERK1/2 and p38 MAPKs. ${ }^{65}$ Resistin also contributes to both the proliferation and migration of VSMC, and its expression is increased after injury in the carotid artery. ${ }^{68}$ For leptin, the evidence is conflicting, depending on the species or disease state. In one study, leptin stimulated the proliferation and migration of rat aortic VSMC in culture, ${ }^{69}$ likely via upregulation of cyclinD1, ERK1/2 and nuclear factor kappa B. ${ }^{70}$ Conversely, 
in a study of the carotid artery from atherosclerotic patients, human VSMC exposed to high concentration of leptin inhibited VSMC growth and downregulation of the leptin receptor, ${ }^{71}$ while leptin also inhibited the angiotensin II-stimulated VSMC proliferation by NOS-dependent mechanisms. ${ }^{29}$ VSMCs are not the only target of PVAT-derived proliferative factors, and for example, adventitial fibroblast growth was shown to be stimulated by complement 3 from PVAT, via c-Jun N-terminal kinase (JNK) activation. ${ }^{72}$ Illustrating the complexity of the regulation of vascular function by PVAT, adiponectin has the opposite effect of decreasing both VSMC growth ${ }^{73,74}$ and insulin-like growth factor-induced migration. ${ }^{75}$

Barandier et $\mathrm{al}^{76}$ showed that cultured media from the PVAT of rat aorta stimulated VSMC growth and without identifying the responsible factors, narrowed the candidates to protein components, as both boiling and proteinase $\mathrm{K}$ decreased this effect. The remaining $40 \%$ of the effect not blocked in these conditions suggested that nonprotein factors may also be involved. For example, free fatty acids, such as oleic acid, have also induced VSMC proliferation. ${ }^{73}$ Steroid hormones, with proven VSMC growth effects, may also help explain this phenomenon, for instance adipocytederived aldosterone was recently demonstrated to stimulate VSMC growth. ${ }^{77,78}$

\section{Influence of PVAT on vascular function in pathophysiological states Obesity, diabetes, and the metabolic syndrome}

Obesity is a chronic disease that has become a world health concern, due to its ever-increasing prevalence. The worldwide obesity pandemic is all the more alarming because of the clear link between obesity and risk for cardiovascular disease and increased mortality. PVAT mass, like total adipose mass, is increased as well in humans with obesity ${ }^{2,59}$ and in all animal models of obesity. ${ }^{39,40,79}$ In humans, this increase in PVAT mass correlates with that of VAT and with the presence of hypertension, insulin resistance, and diabetes. ${ }^{2,54}$

The increase in adipose tissue mass in obesity is supported by both adipocyte expansion and, potentially, proliferation. Alterations in adipose tissue during obesity are far more complex than the simple increase in mass, comprising changes in the composition of lipid droplets, remodeling of adipose ECM, and macrophage/lymphocyte infiltration. A typical finding in obesity is the systemic low-grade inflammation, which is present in the adipose tissue as well, maintaining an imbalance in the secretion of adipokines and promoting oxidative stress, hypoxia, and insulin resistance..$^{80,81}$

It is presumed that alterations in PVAT follow those of adipose tissue elsewhere. However, these processes do not occur simultaneously from the onset of obesity but rather, appear to develop over time in a sequential manner that is not completely understood. Although the anticontractile effects of PVAT are dependent on the mass of PVAT in normal conditions, ${ }^{51}$ in obesity, the unbalanced secretion of PVRFs, together with oxidative stress and macrophage infiltration of PVAT, lead to a widely documented decrease in the anticontractile effect, discussed below. However, this PVAT dysfunction is characteristic of the chronic phase of obesity and may initially be characterized by the opposite, compensatory changes. Early obesity was shown to be accompanied by an increase in the production of NO by mesenteric PVAT, in a mouse model of HFD-induced obesity. ${ }^{42}$ Conversely, with long-term exposure to a HFD, PVAT may contribute to endothelial dysfunction. Removal of PVAT improved endothelial function after 6 months of HFD treatment in rats. In this model, reduced eNOS phosphorylation at the activation site Ser-1177 was associated with decreased AMPK activation and consequently increased mammalian target of rapamycin (mTOR) in aortic tissue. Conversely, a similar expression pattern of MAPK/mTOR was observed in VSMCs cocultured with adipocytes from HFD-treated rats, ${ }^{79}$ suggesting that in obesity, PVAT contributes to the endothelial dysfunction partly via inhibition of the AMPKmediated eNOS activation. In HFD-induced obese mice, similarly to the study above, impaired endothelial function was restored by removing PVAT. ${ }^{39}$ The same was achieved by treatment with ROS scavengers or apocynin. ${ }^{39}$ Evidence from other studies supports the key role of increased ROS production in the development of PVAT dysfunction and loss of anticontractile properties, during obesity. In obese humans, the diminished anticontractile properties of PVAT were restored by treatment with superoxide dismutase (SOD) and catalase, which counteracted the increased ROS production of obese PVAT. ${ }^{59}$ The inhibitory effects of obesity or hypoxia on the anticontractile properties of PVAT was shown to be mimicked by the application of IL-6 or TNF- $\alpha$ and rescued by TNF- $\alpha$ antagonist treatment. ${ }^{59}$ The increased production of ROS was also demonstrated in the New Zealand obese mouse model, where authors showed that SOD expression was decreased and eNOS potentially uncoupled, while PVAT was infiltrated with macrophages. ${ }^{40}$ In a model of metabolic syndrome induced by fructose feeding, the increased markers of oxidative stress and decreased 
expression of antioxidant enzymes were correlated with the increased ratio of mono- to polyunsaturated fatty acids. ${ }^{82}$ Angiotensin-receptor antagonist treatment in the same model restored the increased norepinephrine-induced contraction, in the presence and absence of PVAT. ${ }^{83}$

Macrophage infiltration in adipose tissue has been correlated to the adipocyte lipolysis observed in chronic obesity, suggesting that the release of fatty acids may trigger macrophage infiltration to initiate inflammatory changes in obese adipose tissue. ${ }^{84}$ Besides oxidative stress and macrophage infiltration, another crucial element of PVAT dysfunction in obesity is the alteration in secretion of adipocytokines. As a general rule, circulating levels and tissue production of leptin is increased and of adiponectin decreased, during obesity. This was also observed at the level of PVAT, in HFD models of obesity. ${ }^{4,39,40}$ Despite the increase in leptin release, with obesity, a peripheral resistance to its effects occurs. For example, it was shown in the Zucker rat, that the normal vasorelaxant and $\mathrm{Ca}^{2+}$ inhibitory effect of leptin was lost. ${ }^{85}$ Leptin produced by PVAT was also shown to exacerbate coronary endothelial dysfunction in a model of metabolic syndrome, through increased PKC $\beta$ activation and phosphorylation of eNOS at the inhibitory site Thr-495. ${ }^{86}$

Recent evidence strongly supports the idea that PVAT plays a key role in the development of vascular insulin resistance. It has been shown that the insulin-induced relaxation observed in the presence of PVAT in normal conditions is lost in $\mathrm{db} / \mathrm{db}$ mice when PVAT mass is increased but releases less adiponectin. Additionally, inhibition of JNK restores PVAT-mediated insulin-induced vasorelaxation through adiponectin, which appears to act through AMPK $\alpha 2{ }^{87}$

Although the PVAT dysfunction in models of HFD- or fructose-induced obesity, metabolic syndrome, and type 2 diabetes share many pathophysiological mechanisms and experimental findings, the same may not be true for type 1 diabetes. Thus, Lee et $\mathrm{al}^{88}$ described that acute hyperglycemia and chronic streptozotocin-induced type 1 diabetes led to an increase in the PVRF release and vasorelaxant properties of PVAT.

\section{Hypertension}

The hypertensive vascular dysfunction, characterized by endothelial dysfunction and hypertensive remodeling of the smooth muscle layer, is a well documented process. The complicated mechanisms underlying vascular dysfunction, including a decreased NO bioavailability, activation of the pathways of smooth muscle contraction, vascular oxidative stress, and inflammation, have been investigated in countless studies of animal models of hypertension and clinical studies. By comparison, the role played by PVAT in development of this vascular dysfunction is clearly understudied and to date, there have been no human studies on PVAT in hypertension.

The mass of PVAT, both absolute and relative to body mass, is consistently decreased in rat models of hypertension, such as the spontaneously hypertensive rat (SHR), angiotensin II-infused rats, and deoxycorticosterone acetate (DOCA)-salt rats. ${ }^{61,72,89-91}$ The functional effect of PVAT on smooth muscle contraction was also shown to be altered and the typical anticontractile effect of PVAT diminished or lost. In SHR, this was observed at the aorta and both isolated mesenteric arteries or perfused whole mesenteric bed. ${ }^{61,89,91-94}$ Mechanistically, Gálvez-Prieto et $\mathrm{al}^{91}$ discussed the role of $\mathrm{K}_{\mathrm{V}}$ channels in the PVAT dysfunction in SHR and, using 4-weekold animals, demonstrated that the impairment of PVAT function occurs before the increase in blood pressure. The participation of $\mathrm{K}_{\mathrm{V}} 7$ channels was recently confirmed, where it was seen that $\mathrm{K}_{\mathrm{V}} 7$ blockade abolished the contractile differences between WKY and SHR rats, in the presence of PVAT. ${ }^{93}$ Interestingly, different from what was discussed previously in this review, these authors proposed a diffusion-hindrance effect by PVAT, supported by a decreased sensitivity to both acetylcholine and sodium nitroprusside, in the presence of PVAT. $^{93}$ A recent study reinforced earlier findings by the Lee group, by demonstrating that Mas receptor blockade abolished the PVAT-induced relaxation response, which was diminished in SHR rats compared with WKY rats. ${ }^{94}$ By transferring bath solutions from PVAT-intact vessels and comparing responses in WKY and SHR, these authors and others showed that the decrease in PVAT-induced relaxation observed in SHR rats was not due to the decrease in PVAT mass. ${ }^{92,94}$ This reduction in the anticontractile effects was also not found to be due to the endothelial dysfunction, since it was still present in endothelium-denuded arteries. ${ }^{90}$ The diminished anticontractile effect of PVAT can be restored by atorvastatin treatment, ${ }^{92}$ the same treatment that increased $\mathrm{H}_{2} \mathrm{~S}$ production in PVAT in a previously mentioned study. ${ }^{46}$ Adipokine secretion is also perturbed during hypertension. Leptin secretion is decreased in mesenteric PVAT in SHR rats, ${ }^{89}$ and its effects on angiotensin II-induced contraction and VSMC Ca ${ }^{2+}$ homeostasis are lost. ${ }^{29,95}$ The secretome of aortic PVAT was studied by liquid chromatography-mass spectrometry in a study indicating that the most abundant product secreted by this tissue is complement 3, which induced adventitial fibroblast migration and differentiation via JNK activation. The increased secretion of complement 
3 in DOCA-salt rats correlated with the adventitial remodeling observed in this model of hypertension. ${ }^{72}$

\section{Atherosclerosis}

As the underlying cause of cardiovascular diseases that lead the statistics on human mortality, atherosclerosis is under continuous investigation for mechanisms and therapeutic approaches for prevention and treatment. In view of all the PVAT molecules with potential effects on vessel function (Table 1), it is logical to presume an involvement of this layer in the complex mechanisms of atherosclerosis development. However, the potential link between dysfunction of the PVAT and atherosclerosis, although supported by circumstantial evidence and reasonable hypotheses, has not been studied directly. One of these hypotheses is the theory of "outside to inside" signaling of atherogenesis, ${ }^{96}$ which proposes that events leading to the increased release of proinflammatory and chemotactic mediators in PVAT lead in turn, to the infiltration of macrophages and other immune cells, promoting endothelial dysfunction, hypercoagulability, monocyte adhesion, and plaque formation. ${ }^{96-98}$

There is a scarcity of studies on PVAT in atherosclerosis. In humans, most of these were done in epicardial adipose tissue, which presents the advantage of studying phenomena close to the coronaries but could not be entirely equated to pericoronary PVAT. Increased epicardial adipose tissue size has been correlated to atherosclerotic plaques, the degree of coronary artery stenosis, and cardiovascular events by a number of articles reviewed elsewhere. ${ }^{96}$ In this same tissue, adipokine secretion was found to be unbalanced, such that molecules that promote inflammation and VSMC proliferation, like IL-6, plasminogen activator inhibitor 1 (PAI-1), $\mathrm{TNF} \alpha$, visfatin, and leptin were elevated, whereas adipokines with anti-inflammatory properties, like adiponectin, were decreased. ${ }^{35,99-103}$ Interestingly, the intramyocardial portions of coronary arteries, which lack PVAT, were less likely to develop atherosclerosis. ${ }^{104}$

Human aortic atherosclerosis was correlated with the periaortic PVAT levels of chemerin, visfatin, leptin, and vaspin. ${ }^{105}$ Henrichot et $\mathrm{al}^{35}$ demonstrated that the increased production of IL-8 and MCP-1 in the PVAT of human atherosclerotic aorta was correlated with secretion of these molecules by SAT and associated with macrophage and T-cell infiltration at the interface between PVAT and the adventitia of atherosclerotic aortas. In contrast, peripheral arteries lacking atherosclerosis also lacked these infiltrating cells. Conditioned medium from these human periaortic adipocytes was found to stimulate migration of granulocytes, monocytes, and activated $\mathrm{T}$ cells in vitro. In the apolipoprotein E- knockout mouse model of atherosclerosis, PVAT release of macrophage inflammatory protein $1 \alpha$, IL-1 $\beta$, IL-1 receptor, and IL-6 was increased, and mesenteric PVAT presented macrophage infiltration. ${ }^{106}$ The upregulation in PAI-1, IL-6, and TNF $\alpha$ observed after endovascular balloon injury in mice was abolished by TNF $\alpha$ deletion. ${ }^{107}$

Although these studies demonstrate the detrimental effect of PVAT and its potential involvement in atherosclerosis development, it is an inflamed and dysfunctional PVAT that produces these effects, and the lack of an otherwise normal PVAT may have equally devastating effects. If PVAT is lacking systemically, as it happens in mice lacking PPAR $\gamma$ during preadipocyte development, thermoregulation is impaired, and atherosclerosis development in response to cold-induced activation is exacerbated. ${ }^{108}$

\section{Conclusion}

This review discussed the current evidence for the concept that far from being present only as a mechanical support structure or lipid depot, PVAT possesses the capacity to act in a paracrine manner, on the blood vessel, the function of which it modulates via complex mechanisms (Figure 1). New molecules released from PVAT are constantly being added to the already impressive list of adipokines, cytokines, ROS, lipid species, and gaseous molecules that PVAT produces. The alterations in the anticontractile function of PVAT in obesity, metabolic syndrome, hypertension, or atherosclerosis are correlated with an imbalance in the secretion of adipokines and the presence of inflammation and oxidative stress, leading to vascular dysfunction. The sequence of these processes is unclear, and a key event, common to PVAT dysfunction in cardiovascular disease, appears to be the PVAT infiltration by immune cells that induces the cascade of pathologies, although the trigger for this is not understood. A better understanding of PVAT physiology may allow for the design of therapies for vascular dysfunction and of strategies for directing these therapies to PVAT. An example, based on the different secretory profile of white- or brown-like PVAT, would be treatment that stimulates white-to-brown adipocyte transdifferentiation. However, studies reviewed here also revealed that there are vascular-bed differences in PVAT function that need to be taken into account. Furthermore, more human studies are certainly needed in order to definitively elucidate the role of PVAT in cardiovascular disease.

\section{Disclosure}

The authors report no conflicts of interest in this work. 


\section{References}

1. Schlett CL, Massaro JM, Lehman SJ, et al. Novel measurements of periaortic adipose tissue in comparison to anthropometric measures of obesity, and abdominal adipose tissue. Int J Obes (Lond). 2009;33(2):226-232.

2. Lehman SJ, Massaro JM, Schlett CL, O’Donnell CJ, Hoffmann U, Fox CS. Peri-aortic fat, cardiovascular disease risk factors, and aortic calcification: the Framingham Heart Study. Atherosclerosis. 2010;210(2): 656-661.

3. Fitzgibbons TP, Kogan S, Aouadi M, Hendricks GM, Straubhaar J, Czech MP. Similarity of mouse perivascular and brown adipose tissues and their resistance to diet-induced inflammation. Am J Physiol Heart Circ Physiol. 2011;301(4):H1425-H1437.

4. Chatterjee TK, Stoll LL, Denning GM, et al. Proinflammatory phenotype of perivascular adipocytes: influence of high-fat feeding. Circ Res. 2009;104(4):541-549.

5. Rittig K, Dolderer JH, Balletshofer B, et al. The secretion pattern of perivascular fat cells is different from that of subcutaneous and visceral fat cells. Diabetologia. 2012;55(5):1514-1525.

6. Bell LN, Ward JL, Degawa-Yamauchi M, et al. Adipose tissue production of hepatocyte growth factor contributes to elevated serum HGF in obesity. Am J Physiol Endocrinol Metab. 2006;291(4):E843-E848.

7. Gálvez-Prieto B, Bolbrinker J, Stucchi P, et al. Comparative expression analysis of the renin-angiotensin system components between white and brown perivascular adipose tissue. J Endocrinol. 2008;197(1):55-64.

8. Hansen JB, Kristiansen K. Regulatory circuits controlling white versus brown adipocyte differentiation. Biochem J. 2006;398(2):153-168.

9. Tiraby C, Langin D. Conversion from white to brown adipocytes: a strategy for the control of fat mass? Trends Endocrinol Metab. 2003; 14(10):439-441.

10. Boström P, Wu J, Jedrychowski MP, et al. A PGC1- $\alpha$-dependent myokine that drives brown-fat-like development of white fat and thermogenesis. Nature. 2012;481(7382):463-468.

11. Cao L, Choi EY, Liu X, et al. White to brown fat phenotypic switch induced by genetic and environmental activation of a hypothalamicadipocyte axis. Cell Metab. 2011;14(3):324-338.

12. Mariman EC, Wang P. Adipocyte extracellular matrix composition, dynamics and role in obesity. Cell Mol Life Sci. 2010;67(8): 1277-1292.

13. Giordano A, Song CK, Bowers RR, et al. White adipose tissue lacks significant vagal innervation and immunohistochemical evidence of parasympathetic innervation. Am J Physiol Regul Integr Comp Physiol. 2006;291(5):R1243-R1255.

14. Guzik TJ, Marvar PJ, Czesnikiewicz-Guzik M, Korbut R. Perivascular adipose tissue as a messenger of the brain-vessel axis: role in vascular inflammation and dysfunction. J Physiol Pharmacol. 2007; 58(4):591-610.

15. Scherer PE, Williams S, Fogliano M, Baldini G, Lodish HF. A novel serum protein similar to $\mathrm{C} 1 \mathrm{q}$, produced exclusively in adipocytes. J Biol Chem. 1995;270(45):26746-26749.

16. Arita Y, Kihara S, Ouchi N, et al. Paradoxical decrease of an adiposespecific protein, adiponectin, in obesity. Biochem Biophys Res Commun. 1999;257(1):79-83.

17. Turer AT, Scherer PE. Adiponectin: mechanistic insights and clinical implications. Diabetologia. 2012;55(9):2319-2326.

18. Lam KS, Xu A. Adiponectin: protection of the endothelium. Curr Diab Rep. 2005;5(4):254-259.

19. Ohashi K, Ouchi N, Matsuzawa Y. Adiponectin and hypertension. Am J Hypertens. 2011;24(3):263-269.

20. Okamoto Y, Arita Y, Nishida M, et al. An adipocyte-derived plasma protein, adiponectin, adheres to injured vascular walls. Horm Metab Res. 2000;32(2):47-50.

21. Tilg H, Moschen AR. Adipocytokines: mediators linking adipose tissue, inflammation and immunity. Nat Rev Immunol. 2006;6(10): $772-783$.

22. Fésüs G, Dubrovska G, Gorzelniak K, et al. Adiponectin is a novel humoral vasodilator. Cardiovasc Res. 2007;75(4):719-727.
23. Zhu W, Cheng KK, Vanhoutte PM, Lam KS, Xu A. Vascular effects of adiponectin: molecular mechanisms and potential therapeutic intervention. Clin Sci (Lond). 2008;114(5):361-374.

24. Mohammed MM, Myers DS, Sofola OA, Hainsworth R, Drinkhill MJ. Vasodilator effects of leptin on canine isolated mesenteric arteries and veins. Clin Exp Pharmacol Physiol. 2007;34(8):771-774.

25. Nakagawa K, Higashi Y, Sasaki S, Oshima T, Matsuura H, Chayama K. Leptin causes vasodilation in humans. Hypertens Res. 2002;25(2):161-165.

26. Sahin AS, Bariskaner H. The mechanisms of vasorelaxant effect of leptin on isolated rabbit aorta. Fundam Clin Pharmacol. 2007;21(6): 595-600.

27. Kimura K, Tsuda K, Baba A, et al. Involvement of nitric oxide in endothelium-dependent arterial relaxation by leptin. Biochem Biophys Res Commun. 2000;273(2):745-749.

28. Rahmouni K, Haynes WG. Endothelial effects of leptin: implications in health and diseases. Curr Diab Rep. 2005;5(4):260-266.

29. Rodríguez A, Fortuño A, Gómez-Ambrosi J, Zalba G, Diez J, Frühbeck G. The inhibitory effect of leptin on angiotensin II-induced vasoconstriction in vascular smooth muscle cells is mediated via a nitric oxide-dependent mechanism. Endocrinology. 2007;148(1): 324-331.

30. Knudson JD, Dincer UD, Zhang C, et al. Leptin receptors are expressed in coronary arteries, and hyperleptinemia causes significant coronary endothelial dysfunction. Am J Physiol Heart Circ Physiol. 2005;289(1): H48-H56.

31. Shek EW, Brands MW, Hall JE. Chronic leptin infusion increases arterial pressure. Hypertension. 1998;31(1 Pt 2):409-414.

32. Cao R, Brakenhielm E, Wahlestedt C, Thyberg J, Cao Y. Leptin induces vascular permeability and synergistically stimulates angiogenesis with FGF-2 and VEGF. Proc Natl Acad Sci U S A. 2001;98(11): 6390-6395.

33. Yamawaki H, Tsubaki N, Mukohda M, Okada M, Hara Y. Omentin, a novel adipokine, induces vasodilation in rat isolated blood vessels. Biochem Biophys Res Commun. 2010;393(4):668-672.

34. Lee YC, Chang HH, Chiang CL, et al. Role of perivascular adipose tissue-derived methyl palmitate in vascular tone regulation and pathogenesis of hypertension. Circulation. 2011;124(10):1160-1171.

35. Henrichot E, Juge-Aubry CE, Pernin A, et al. Production of chemokines by perivascular adipose tissue: a role in the pathogenesis of atherosclerosis? Arterioscler Thromb Vasc Biol. 2005;25(12): 2594-2599.

36. Gao YJ, Lu C, Su LY, Sharma AM, Lee RM. Modulation of vascular function by perivascular adipose tissue: the role of endothelium and hydrogen peroxide. Br J Pharmacol. 2007;151(3):323-331.

37. Gao YJ, Takemori K, Su LY, et al. Perivascular adipose tissue promotes vasoconstriction: the role of superoxide anion. Cardiovasc Res. 2006;71(2):363-373.

38. Li Y, Mihara K, Saifeddine M, et al. Perivascular adipose tissue-derived relaxing factors: release by peptide agonists via proteinase-activated receptor-2 (PAR2) and non-PAR2 mechanisms. Br J Pharmacol. 2011;164(8):1990-2002.

39. Ketonen J, Shi J, Martonen E, Mervaala E. Periadventitial adipose tissue promotes endothelial dysfunction via oxidative stress in diet-induced obese C57Bl/6 mice. Circ J. 2010;74(7):1479-1487.

40. Marchesi C, Ebrahimian T, Angulo O, Paradis P, Schiffrin EL. Endothelial nitric oxide synthase uncoupling and perivascular adipose oxidative stress and inflammation contribute to vascular dysfunction in a rodent model of metabolic syndrome. Hypertension. 2009;54(6): 1384-1392.

41. Dashwood MR, Dooley A, Shi-Wen X, Abraham DJ, Souza DS. Does periadventitial fat-derived nitric oxide play a role in improved saphenous vein graft patency in patients undergoing coronary artery bypass surgery? J Vasc Res. 2007;44(3):175-181.

42. Gil-Ortega M, Stucchi P, Guzmán-Ruiz R, et al. Adaptative nitric oxide overproduction in perivascular adipose tissue during early diet-induced obesity. Endocrinology. 2010;151(7):3299-3306. 
43. Malinowski M, Deja MA, Golba KS, Roleder T, Biernat J, Woś S. Perivascular tissue of internal thoracic artery releases potent nitric oxide and prostacyclin-independent anticontractile factor. Eur J Cardiothorac Surg. 2008;33(2):225-231.

44. Fang L, Zhao J, Chen Y, et al. Hydrogen sulfide derived from periadventitial adipose tissue is a vasodilator. J Hypertens. 2009;27(11):2174-2185.

45. Köhn C, Schleifenbaum J, Szijártó IA, et al. Differential effects of cystathionine- $\gamma$-lyase-dependent vasodilatory $\mathrm{H} 2 \mathrm{~S}$ in periadventitial vasoregulation of rat and mouse aortas. PLoS One. 2012;7(8):e41951.

46. Wójcicka G, Jamroz-Wiśniewska A, Atanasova P, Chaldakov GN, Chylińska-Kula B, Beltowski J. Differential effects of statins on endogenous $\mathrm{H} 2 \mathrm{~S}$ formation in perivascular adipose tissue. Pharmacol Res. 2010;63(1):68-76.

47. Lee RM, Bader M, Alenina N, Santos RA, Gao YJ, Lu C. Mas receptors in modulating relaxation induced by perivascular adipose tissue. Life Sci. 2011;89(13-14):467-472.

48. Soltis EE, Cassis LA. Influence of perivascular adipose tissue on rat aortic smooth muscle responsiveness. Clin Exp Hypertens A. 1991;13(2):277-296.

49. Dubrovska G, Verlohren S, Luft FC, Gollasch M. Mechanisms of ADRF release from rat aortic adventitial adipose tissue. Am J Physiol Heart Circ Physiol. 2004;286(3):H1107-H1113.

50. Löhn M, Dubrovska G, Lauterbach B, Luft FC, Gollasch M, Sharma AM. Periadventitial fat releases a vascular relaxing factor FASEB J. 2002;16(9):1057-1063.

51. Verlohren S, Dubrovska G, Tsang SY, et al. Visceral periadventitial adipose tissue regulates arterial tone of mesenteric arteries. Hypertension. 2004;44(3):271-276

52. Gollasch M, Dubrovska G. Paracrine role for periadventitial adipose tissue in the regulation of arterial tone. Trends Pharmacol Sci. 2004;25(12):647-653.

53. Reifenberger MS, Turk JR, Newcomer SC, Booth FW, Laughlin MH. Perivascular fat alters reactivity of coronary artery: effects of diet and exercise. Med Sci Sports Exerc. 2007;39(12):2125-2134.

54. Rittig K, Staib K, Machann J, et al. Perivascular fatty tissue at the brachial artery is linked to insulin resistance but not to local endothelial dysfunction. Diabetologia. 2008;51(11):2093-2099.

55. Lu C, Zhao AX, Gao YJ, Lee RM. Modulation of vein function by perivascular adipose tissue. Eur J Pharmacol. 2011;657(1-3):111-116.

56. Lynch FM, Withers SB, Yao Z, et al. Perivascular adipose tissuederived adiponectin activates $\mathrm{BKCa}$ channels to induce anticontractile responses. Am J Physiol Heart Circ Physiol. Epub January 4, 2013.

57. Gao YJ, Zeng ZH, Teoh K, et al. Perivascular adipose tissue modulates vascular function in the human internal thoracic artery. J Thorac Cardiovasc Surg. 2005;130(4):1130-1136.

58. Withers SB, Agabiti-Rosei C, Livingstone DM, et al. Macrophage activation is responsible for loss of anticontractile function in inflamed perivascular fat. Arterioscler Thromb Vasc Biol. 2011;31(4): 908-913

59. Greenstein AS, Khavandi K, Withers SB, et al. Local inflammation and hypoxia abolish the protective anticontractile properties of perivascular fat in obese patients. Circulation. 2009;119(12):1661-1670.

60. Lee RM, Lu C, Su LY, Gao YJ. Endothelium-dependent relaxation factor released by perivascular adipose tissue. J Hypertens. 2009; 27(4):782-790.

61. Gálvez-Prieto B, Somoza B, Gil-Ortega M, et al. Anticontractile effect of perivascular adipose tissue and leptin are reduced in hypertension. Front Pharmacol. 2012;3:103.

62. Lobato NS, Neves KB, Filgueira FP, et al. The adipokine chemerin augments vascular reactivity to contractile stimuli via activation of the MEK-ERK1/2 pathway. Life Sci. 2012;91(13-14):600-606.

63. Yamawaki H. Vascular effects of novel adipocytokines: focus on vascular contractility and inflammatory responses. Biol Pharm Bull. 2011;34(3):307-310.

64. Maenhaut N, Boydens C, Van de Voorde J. Hypoxia enhances the relaxing influence of perivascular adipose tissue in isolated mice aorta. Eur J Pharmacol. 2010;641(2-3):207-212.
65. Wang P, Xu TY, Guan YF, Su DF, Fan GR, Miao CY. Perivascular adipose tissue-derived visfatin is a vascular smooth muscle cell growth factor: role of nicotinamide mononucleotide. Cardiovasc Res. 2009;81(2):370-380.

66. Lu C, Su LY, Lee RM, Gao YJ. Mechanisms for perivascular adipose tissue-mediated potentiation of vascular contraction to perivascular neuronal stimulation: the role of adipocyte-derived angiotensin II. Eur J Pharmacol. 2010;634(1-3):107-112.

67. Payne GA, Bohlen HG, Dincer UD, Borbouse L, Tune JD. Periadventitial adipose tissue impairs coronary endothelial function via PKC-beta-dependent phosphorylation of nitric oxide synthase. Am J Physiol Heart Circ Physiol. 2009;297(1):H460-H465.

68. Shyu KG, Lien LM, Wang BW, Kuan P, Chang H. Resistin contributes to neointimal formation via oxidative stress after vascular injury. Clin Sci (Lond). 2011;120(3):121-129.

69. Oda A, Taniguchi T, Yokoyama M. Leptin stimulates rat aortic smooth muscle cell proliferation and migration. Kobe J Med Sci. 2001;47(3): $141-150$.

70. Huang F, Xiong X, Wang H, You S, Zeng H. Leptin-induced vascular smooth muscle cell proliferation via regulating cell cycle, activating ERK1/2 and NF-kappaB. Acta Biochim Biophys Sin (Shanghai). 2010;42(5):325-331.

71. Bohlen F, Kratzsch J, Mueller M, et al. Leptin inhibits cell growth of human vascular smooth muscle cells. Vascul Pharmacol. 2007;46(1): 67-71.

72. Ruan CC, Zhu DL, Chen QZ, et al. Perivascular adipose tissue-derived complement 3 is required for adventitial fibroblast functions and adventitial remodeling in deoxycorticosterone acetate-salt hypertensive rats. Arterioscler Thromb Vasc Biol. 2010;30(12):2568-2574.

73. Lamers D, Schlich R, Greulich S, Sasson S, Sell H, Eckel J. Oleic acid and adipokines synergize in inducing proliferation and inflammatory signaling in human vascular smooth muscle cells. J Cell Mol Med. 2011;15(5):1177-1188.

74. Wang Y, Lam KS, Xu JY, et al. Adiponectin inhibits cell proliferation by interacting with several growth factors in an oligomerization-dependent manner. J Biol Chem. 2005;280(18):18341-18347.

75. Motobayashi Y, Izawa-Ishizawa Y, Ishizawa K, et al. Adiponectin inhibits insulin-like growth factor-1-induced cell migration by the suppression of extracellular signal-regulated kinase 1/2 activation, but not Akt in vascular smooth muscle cells. Hypertens Res. 2009;32(3):188-193.

76. Barandier C, Montani JP, Yang Z. Mature adipocytes and perivascular adipose tissue stimulate vascular smooth muscle cell proliferation: effects of aging and obesity. Am J Physiol Heart Circ Physiol. 2005;289(5):H1807-H1813.

77. Briones AM, Nguyen Dinh Cat A, Callera GE, et al. Adipocytes produce aldosterone through calcineurin-dependent signaling pathways: implications in diabetes mellitus-associated obesity and vascular dysfunction. Hypertension. 2012;59(5):1069-1078.

78. Nguyen Dinh Cat A, Briones AM, Callera GE, et al. Adipocyte-derived factors regulate vascular smooth muscle cells through mineralocorticoid and glucocorticoid receptors. Hypertension. 2011;58(3):479-488.

79. Ma L, Ma S, He H, et al. Perivascular fat-mediated vascular dysfunction and remodeling through the AMPK/m TOR pathway in high-fat diet-induced obese rats. Hypertens Res. 2010;33(5):446-453.

80. Weisberg SP, McCann D, Desai M, Rosenbaum M, Leibel RL, Ferrante AW Jr. Obesity is associated with macrophage accumulation in adipose tissue. J Clin Invest. 2003;112(12):1796-1808.

81. Xu H, Barnes GT, Yang Q, et al. Chronic inflammation in fat plays a crucial role in the development of obesity-related insulin resistance. J Clin Invest. 2003;112(12):1821-1830.

82. Rebolledo A, Rebolledo OR, Marra CA, et al. Early alterations in vascular contractility associated to changes in fatty acid composition and oxidative stress markers in perivascular adipose tissue. Cardiovasc Diabetol. 2010;9(1):65.

83. Huang F, Lezama MA, Ontiveros JA, et al. Effect of losartan on vascular function in fructose-fed rats: the role of perivascular adipose tissue. Clin Exp Hypertens. 2010;32(2):98-104. 
84. Kosteli A, Sugaru E, Haemmerle G, et al. Weight loss and lipolysis promote a dynamic immune response in murine adipose tissue. J Clin Invest. 2010;120(10):3466-3479.

85. Fortuño A, Rodríguez A, Gómez-Ambrosi J, et al. Leptin inhibits angiotensin II-induced intracellular calcium increase and vasoconstriction in the rat aorta. Endocrinology. 2002;143(9):3555-3560.

86. Payne GA, Borbouse L, Kumar S, et al. Epicardial perivascular adipose-derived leptin exacerbates coronary endothelial dysfunction in metabolic syndrome via a protein kinase C-beta pathway. Arterioscler Thromb Vasc Biol. 2010;30(9):1711-1717.

87. Meijer RI, Bakker W, Alta CL, et al. Perivascular adipose tissue control of insulin-induced vasoreactivity in muscle is impaired in $\mathrm{db} / \mathrm{db}$ mice. Diabetes. 2013;62(2):590-598.

88. Lee RM, Lu C, Su LY, Werstuck G, Gao YJ. Effects of hyperglycemia on the modulation of vascular function by perivascular adipose tissue. J Hypertens. 2009;27(1):118-131.

89. Gálvez B, de Castro J, Herold D, et al. Perivascular adipose tissue and mesenteric vascular function in spontaneously hypertensive rats. Arterioscler Thromb Vasc Biol. 2006;26(6):1297-1302.

90. Lee RM, Ding L, Lu C, Su LY, Gao YJ. Alteration of perivascular adipose tissue function in angiotensin II-induced hypertension. Can J Physiol Pharmacol. 2009;87(11):944-953.

91. Gálvez-Prieto B, Dubrovska G, Cano MV, et al. A reduction in the amount and anti-contractile effect of periadventitial mesenteric adipose tissue precedes hypertension development in spontaneously hypertensive rats. Hypertens Res. 2008;31(7):1415-1423.

92. Zeng $\mathrm{ZH}$, Zhang $\mathrm{ZH}$, Luo $\mathrm{BH}$, et al. The functional changes of the perivascular adipose tissue in spontaneously hypertensive rats and the effects of atorvastatin therapy. Clin Exp Hypertens. 2009;31(4): 355-363.

93. Li R, Andersen I, Aleke J, Golubinskaya V, Gustafsson H, Nilsson H. Reduced anti-contractile effect of perivascular adipose tissue on mesenteric small arteries from spontaneously hypertensive rats: role of $\mathrm{Kv} 7$ channels. Eur J Pharmacol. 2013;698(1-3):310-315.

94. Lu C, Su LY, Lee RM, Gao YJ. Alterations in perivascular adipose tissue structure and function in hypertension. Eur $J$ Pharmacol. 2011;656(1-3):68-73.

95. Rodríguez A, Frühbeck G, Gómez-Ambrosi J, et al. The inhibitory effect of leptin on angiotensin II-induced vasoconstriction is blunted in spontaneously hypertensive rats. J Hypertens. 2006;24(8):1589-1597.

96. Verhagen SN, Visseren FL. Perivascular adipose tissue as a cause of atherosclerosis. Atherosclerosis. 2011;214(1):3-10.
97. Thalmann S, Meier CA. Local adipose tissue depots as cardiovascular risk factors. Cardiovasc Res. 2007;75(4):690-701.

98. Vela D, Buja LM, Madjid M, et al. The role of periadventitial fat in atherosclerosis. Arch Pathol Lab Med. 2007;131(3):481-487.

99. Baker AR, Silva NF, Quinn DW, et al. Human epicardial adipose tissue expresses a pathogenic profile of adipocytokines in patients with cardiovascular disease. Cardiovasc Diabetol. 2006;5:1.

100. Cheng KH, Chu CS, Lee KT, et al. Adipocytokines and proinflammatory mediators from abdominal and epicardial adipose tissue in patients with coronary artery disease. Int J Obes (Lond). 2008;32(2): 268-274.

101. Eiras S, Teijeira-Fernández E, Shamagian LG, Fernandez AL, Vazquez-Boquete A, Gonzalez-Juanatey JR. Extension of coronary artery disease is associated with increased IL-6 and decreased adiponectin gene expression in epicardial adipose tissue. Cytokine. 2008; 43(2):174-180.

102. Mazurek T, Zhang L, Zalewski A, et al. Human epicardial adipose tissue is a source of inflammatory mediators. Circulation. 2003;108(20): 2460-2466.

103. Silaghi A, Achard V, Paulmyer-Lacroix O, et al. Expression of adrenomedullin in human epicardial adipose tissue: role of coronary status. Am J Physiol Endocrinol Metab. 2007;293(5):E1443-E1450.

104. Ishikawa Y, Akasaka Y, Ito K, et al. Significance of anatomical properties of myocardial bridge on atherosclerosis evolution in the left anterior descending coronary artery. Atherosclerosis. 2006;186(2):380-389.

105. Spiroglou SG, Kostopoulos CG, Varakis JN, Papadaki HH. Adipokines in periaortic and epicardial adipose tissue: differential expression and relation to atherosclerosis. J Atheroscler Thromb. 2010;17(2): 115-130.

106. Lohmann C, Schäfer N, von Lukowicz T, et al. Atherosclerotic mice exhibit systemic inflammation in periadventitial and visceral adipose tissue, liver, and pancreatic islets. Atherosclerosis. 2009;207(2): 360-367.

107. Takaoka M, Suzuki H, Shioda S, et al. Endovascular injury induces rapid phenotypic changes in perivascular adipose tissue. Arterioscler Thromb Vasc Biol. 2010;30(8):1576-1582.

108. Chang L, Villacorta L, Li R, et al. Loss of perivascular adipose tissue on peroxisome proliferator-activated receptor- $\gamma$ deletion in smooth muscle cells impairs intravascular thermoregulation and enhances atherosclerosis. Circulation. 2012;126(9):1067-1078.
Vascular Health and Risk Management

\section{Publish your work in this journal}

Vascular Health and Risk Management is an international, peerreviewed journal of therapeutics and risk management, focusing on concise rapid reporting of clinical studies on the processes involved in the maintenance of vascular health; the monitoring, prevention and treatment of vascular disease and its sequelae; and the involvement of

\section{Dovepress}

metabolic disorders, particularly diabetes. This journal is indexed on PubMed Central and MedLine. The manuscript management system is completely online and includes a very quick and fair peer-review system, which is all easy to use. Visit http://www.dovepress.com/ testimonials.php to read real quotes from published authors. 